\title{
Study of Circulation Drive in High-Speed Rotating Flow Field
}

\author{
Xu Fan ${ }^{1}$, and Bo $\operatorname{Ran}^{1}$ \\ ${ }^{1}$ Gas Dynamics Sciences, Research Department, 300180 Tianjin, China
}

\begin{abstract}
In the rotor with high speed, there is a certain axial circulation in the internal gas, which is necessary to analyze the cause of formation and influence factors for understanding better the internal flow field. There are many factors affecting the axial circulation. Different circulation drives have different effects on the flow field. In this paper, numerical computation with N-S equations is used to compute the flow field parameters and analyze the mechanism of the flow field. The influence of the temperature of the end cap on the flow field is mainly disscussed. By comparing and analyzing the streamline shape and the size of vortex region under different temperature drive, an effective method is provided for the study of axial circulation in the highspeed rotating flow field.
\end{abstract}

\section{Introduction}

In the rotor with high speed, the gas entering the rotor will not only make circular motion along with the rotor. In addition, due to the influence of rarefied gas state and boundary barrier, it will also form axial circulation inside the rotor, making the flow field complex and difficult to analyze. Sometimes a certain axial circulation is also needed to accelerate the flow of gas in the rotor. Therefore, it is an important subject for the study of the high-speed rotating flow field to analyze the production and influence factors of the axial circulation with an effective method [1-3].

There are many factors affecting the axial circulation. Different circulation drives have different effects on the flow field. Among these factors, the influence of temperature on the axial circulation is especially obvious. The temperature difference between the different parts of the rotor, such as the top and bottom end cap and the wall, directly affects the flow state of the internal gas. Therefore, it is of great significance to study the influence of the temperature drive on axial circulation at different parts of the rotor for analysis of the nature of the flow field.

In this paper, numerical computation with N-S equations is used to compute the flow field parameters [4] and analyze the streamline diagram and the mechanism of the flow field. The influence of the temperature of the end cap on the flow field is mainly disscussed. By comparing and analyzing the streamline shape and the size of vortex region under different temperature drive, an effective method is provided for the study of axial circulation in high-speed rotating flow field.

\section{Physical model and numerical calculation}

\subsection{Physical model}

As shown in Fig. 1. This is a physical model for a highspeed rotating flow field.

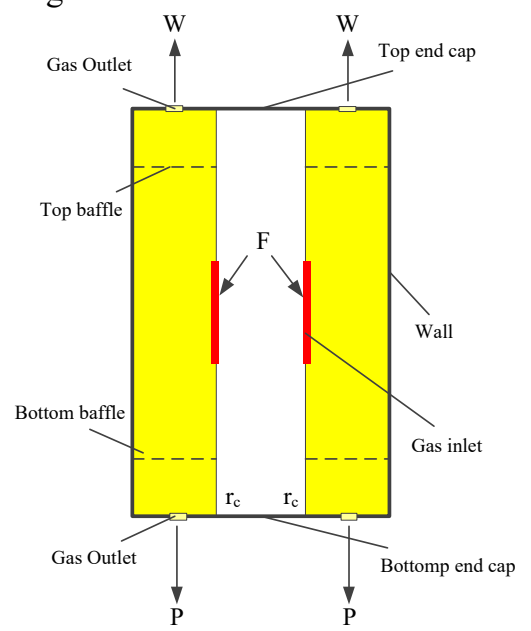

Fig. 1. Schematic diagram of physical model.

Due to the high-speed rotation of the rotor, the gas pressure in the rotor is exponentially distributed along the radial direction.. The Knudsen number is equal to 1 in the transition region, and the region is greater than 1 is the viscous flow region, which is less than 1 is the molecular flow region. It is difficult to calculate the flow field simultaneously in the transition region, the viscous flow region and the molecular flow region [5-6]. By 
calculating, the Kn number is 1 when $r_{c}$ equals 0.7 at a given speed, where the inner boundary is set. This paper only calculates the viscous flow region between the inner boundary and the wall. In the physical model, the gas inlet is set at the inner boundary, and the gas $\mathrm{F}$ enters from the side of the cylinder in the entrance section. A narrow annular slit is made at the top and bottom end cap to simulate the gas outlet. The gas F flows out from the two outlets forming the gas $\mathrm{P}$ and $\mathrm{W}$. It is assumed that the gas velocity near the inlet is uniformly distributed along the axial direction and the gas velocity near the slit is uniformly distributed along the radical direction. There are top and bottom baffles in the rotor. Assuming that the top and bottom baffles are infinitely thin, annular holes are used to simulate the openings of baffles. The location and size of the annular holes are the same as those of the actual baffle holes.

\subsection{Numerical calculation}

The basic assumptions made in the N-S equations are:

(1) Continuous medium hypothesis;

(2) The fluid is an isotropic Newtonian fluid, and Fourier law is satisfied in the heat transfer property;

(3) The fluid satisfies the ideal gas equation of state and is in the state of thermodynamic equilibrium;

(4) Mass force is limited to gravity;

(5) The Stokes hypothesis is used for the expansion viscosity coefficient of compressible fluid, making the expansion viscosity coefficient be $\lambda=-2 \mu / 3$;

Under the above assumptions, the equations in the cylindrical coordinate system are expressed as follows [7]:

Continuous equation:

$$
\frac{\partial \rho}{\partial \mathrm{t}}+\frac{1}{r} \frac{\partial}{\partial r}\left(\mathrm{r} \rho \mathrm{V}_{r}\right)+\frac{1}{r} \frac{\partial}{\partial \theta}\left(\rho \mathrm{V}_{\theta}\right)+\frac{\partial}{\partial z}\left(\rho \mathrm{V}_{z}\right)=0
$$

Momentum equation:

Radial direction:

$$
\begin{aligned}
\rho\left(\frac{D V_{\mathrm{r}}}{D \mathrm{t}}-\frac{V_{\theta}^{2}}{r}\right)= & \rho g_{r}-\frac{\partial p}{\partial r}+\frac{\partial}{\partial r}\left[2 \mu \frac{\partial V_{r}}{\partial r}-\frac{2}{3} \mu(\nabla \cdot \stackrel{\sigma}{V})\right]+ \\
& \frac{1}{r} \frac{\partial}{\partial \theta}\left[\mu\left(\frac{1}{r} \frac{\partial V_{r}}{\partial \theta}+\frac{\partial V_{\theta}}{\partial r}-\frac{V_{\theta}}{r}\right)\right]+ \\
& \frac{\partial}{\partial z}\left[\mu\left(\frac{\partial V_{r}}{\partial z}+\frac{\partial V_{z}}{\partial r}\right)\right]+\frac{2 \mu}{r}\left(\frac{\partial V_{r}}{\partial r}-\frac{1}{r} \frac{\partial V_{\theta}}{\partial \theta}-\frac{V_{r}}{r}\right)
\end{aligned}
$$

Angular direction:

$$
\begin{aligned}
\rho\left(\frac{D V_{\theta}}{D \mathrm{t}}+\frac{V_{r} V_{\theta}}{r}\right) & =\rho g_{\theta}-\frac{1}{r} \frac{\partial p}{\partial \theta}+\frac{1}{\mathrm{r}} \frac{\partial}{\partial \theta}\left[\frac{2 \mu}{r} \frac{\partial V_{\theta}}{\partial \theta}-\frac{2}{3} \mu(\nabla \cdot \stackrel{\varpi}{V})\right]+ \\
& \frac{\partial}{\partial z}\left[\mu\left(\frac{1}{r} \frac{\partial V_{z}}{\partial \theta}+\frac{\partial V_{\theta}}{\partial z}\right)\right]+\frac{\partial}{\partial r}\left[\mu\left(\frac{1}{r} \frac{\partial V_{r}}{\partial \theta}+\frac{\partial V_{\theta}}{\partial r}-\frac{V_{\theta}}{r}\right)\right] \\
& +\frac{2 \mu}{r}\left(\frac{2}{r} \frac{\partial V_{r}}{\partial \theta}+\frac{\partial V_{\theta}}{\partial r}-\frac{V_{\theta}}{r}\right)
\end{aligned}
$$

Axial direction:

$$
\begin{aligned}
\rho \frac{D V_{z}}{D \mathrm{t}}= & \rho g_{z}-\frac{\partial p}{\partial z}+\frac{1}{\mathrm{r}} \frac{\partial}{\partial r}\left[\mu r\left(\frac{\partial V_{z}}{\partial r}+\frac{\partial V_{r}}{\partial z}\right)\right]+ \\
& \frac{1}{r} \frac{\partial}{\partial \theta}\left[\mu\left(\frac{1}{r} \frac{\partial V_{z}}{\partial \theta}+\frac{\partial V_{\theta}}{\partial z}\right)\right]+\frac{\partial}{\partial z}\left[2 \mu \frac{\partial V_{z}}{\partial z}-\frac{2}{3} \mu(\nabla \cdot V)\right]
\end{aligned}
$$

Energy equation:

$$
\begin{aligned}
\rho \frac{D\left(C_{P} T\right)}{D \mathrm{t}}= & \frac{D p}{D t}+\Phi+\frac{1}{\mathrm{r}} \frac{\partial}{\partial r}\left(\kappa \mathrm{r} \frac{\partial T}{\partial r}\right)+\frac{1}{r^{2}} \frac{\partial}{\partial \theta}\left(\kappa \frac{\partial T}{\partial \theta}\right) \\
& +\frac{\partial}{\partial z}\left(\kappa \frac{\partial T}{\partial z}\right)+\frac{\partial Q}{\partial t}
\end{aligned}
$$

State equation:

$$
M \mathrm{p}=\rho R T
$$

In the equations, the column coordinate radial, angular and axial coordinates are marked by $r, \theta, z$. The velocity component in the direction of $r, \theta, z$ are marked by $V_{r}, V_{\theta}, V_{z}$. The static pressure of gas is marked by $\mathrm{p}$. The gas temperature is marked by $T$. The gas density is marked by $\rho$. The dynamic viscosity coefficient of gas is marked by $\mu$. The specific heat at constant pressure of gas is marked by $C_{P}$. The coefficient of gas heat conductivity is marked by $\kappa$. The molecular weight of gas is marked by $M$. The universal gas constant is marked by $R$. The viscous dissipation coefficient is marked by $\Phi$. The unit volume calorific value of heat source in the flow field is marked by $Q$.

In the numerical calculation process, this paper linearize the N-S equations and make them dimensionless, then use the finite difference method to discretize the dimensionless equations, so as to get the fluid parameter values of each cell [8].

\section{Calculation results and discussion}

\subsection{Parameter setting}

According to the above caculation method, the drive quantities are set which affect the axial circulation. In the physical model, the driving mechanisms that can affect the axial circulation mainly include the top and bottom mechanical drive, the wall thermal drive, the gas inlet radial, angular and axial drive. The parameters of each drive are set as shown in Table 1.

Table 1. The parameters of each drive setting.

\begin{tabular}{|c|c|}
\hline Drive Quantity & Value \\
\hline $\begin{array}{c}\text { The top mechanical } \\
\text { drive quantity }\end{array}$ & -0.11 \\
\hline $\begin{array}{c}\text { The bottom mechanical } \\
\text { drive quantity }\end{array}$ & -0.001 \\
\hline The wall thermal drive quantity & $0 \sim 0.03$ \\
\hline $\begin{array}{c}\text { The gas inlet radial } \\
\text { drive quantity }\end{array}$ & 0.60 \\
\hline $\begin{array}{c}\text { The gas inlet angular } \\
\text { drive quantity }\end{array}$ & -0.01 \\
\hline
\end{tabular}




\begin{tabular}{|c|c|}
\hline $\begin{array}{c}\text { The gas inlet angular } \\
\text { drive quantity }\end{array}$ & 0.16 \\
\hline
\end{tabular}

\subsection{Streamlines study}

According to Tab. 1, the wall thermal drive quantity is assumed as 0.02 , and fully driving streamlines are shown in Fig. 2.

From the streamlines shown in Fig. 2, it is obvious that under the common effect of mechanical drive, wall thermal drive and gas inlet drive, there are three large vortexes in the flow field. As shown in the diagram, the vortex $I$ is near the axial position of the gas inlet, the vortex II is close to the wall, and the vortex III is in the top baffle nearby. In addition, there are three small vortexes between the baffle and the end cap. The influence of the vortex produced in the flow field on the axial caculation is defferent, and further analysis is needed to draw a conclusion.

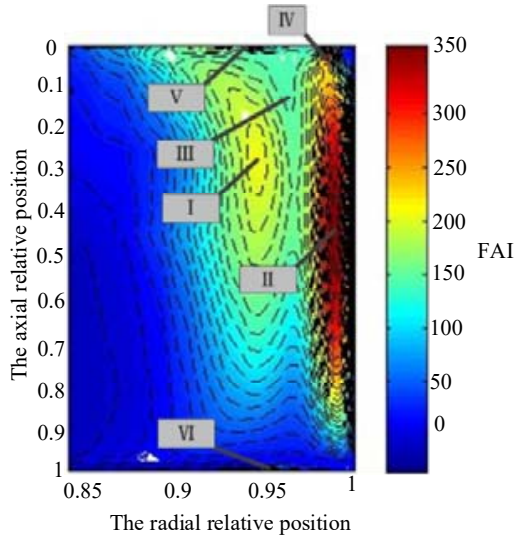

Fig. 2. Fully driving streamlines.

A streamline is a connection of the equivalent points of a flow function, the direction of whose tangent line coincides with the direction of the velocity vector. It is necessary to judge the positive and negative of the mass flow through the calculation of the flow field, and obtain the direction of the vortex. Based on that, the influence of each vortex on the axial circulation can be inferred. Fig. 3 is the section flow pattern with full drive.

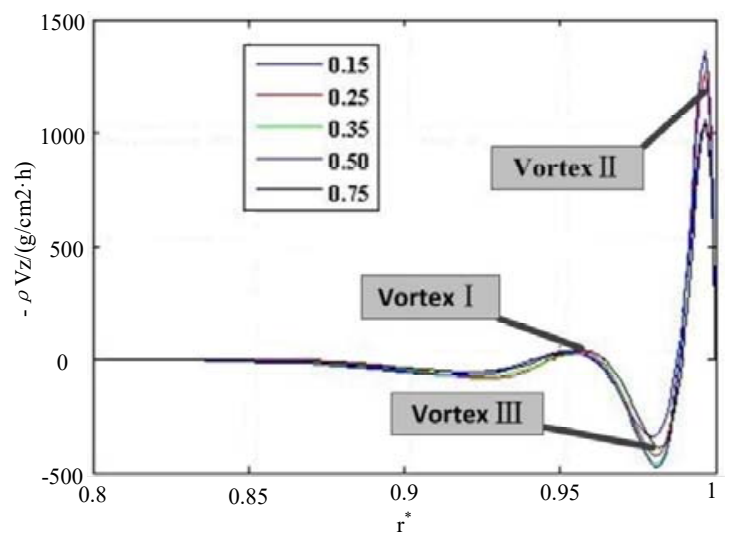

Fig. 3. The section flow pattern with full drive.

According to the positive and negative of mass flow, it can be judged that the vortex I and II are counter clockwise, which can be in favor of the axial circulation in the rotor. While the vortex III is clockwise, the effect is the opposite. Based on the effect of the above vortex on the axial circulation, it is possible to further analyze the effect of temperature difference between the top and bottom end cap on vortex in the internal flow field of the rotor.

The temperature difference between the top and bottom end cap directly affects the temperature of the wall. Therefore, it is necessary to set the wall thermal drive to 0 , then comparing the simulated streamlines with the streamlines under the full drive mode and observing the change of vortexes. The missing vortex is produced by the effect of the wall thermal drive.

Fig. 4 is the streamlines without the wall thermal drive. Compared with Fig. 2, Fig. 4 clearly lacks the vortex II, while other vortex streamlines have little change. It is possible to judge that the vortex II is mainly produced by the wall thermal drive. From Fig. 3, the existence of vortex II can promote the axial circulation, so the wall thermal drive is needed to adjust and find the best value, so as to maximize the promotion.

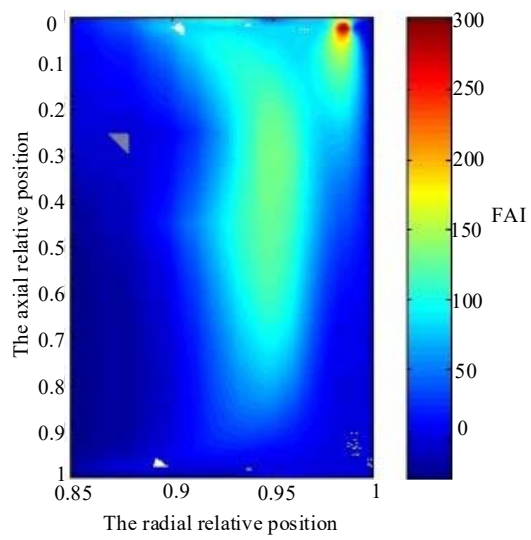

Fig. 4. The streamlines without the wall thermal drive.

\subsection{Effect of different end cap temperature}

Fig. 5 to Fig. 7 represent respectively the streamlines of the wall thermal drive quantity with $0.01,0.02$ and 0.03 . 


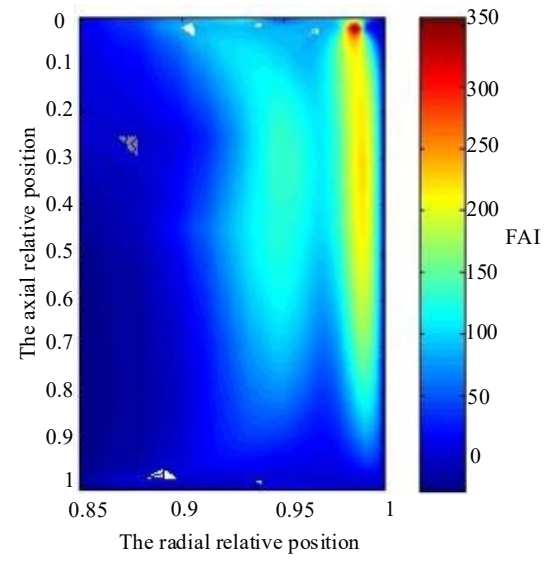

Fig. 5. The streamlines of the wall thermal drive quantity with 0.01 .

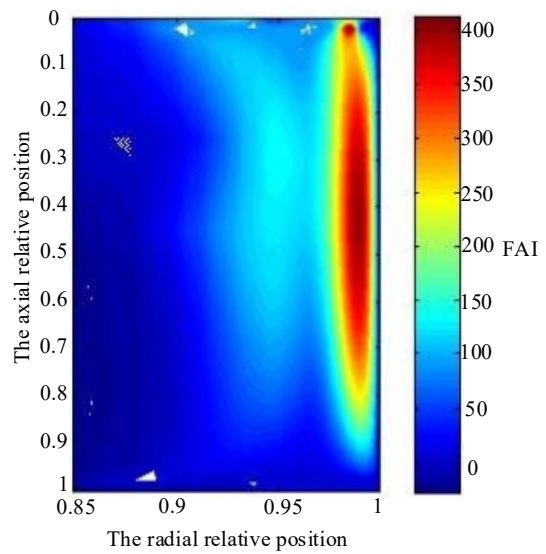

Fig. 6. The streamlines of the wall thermal drive quantity with 0.02 .

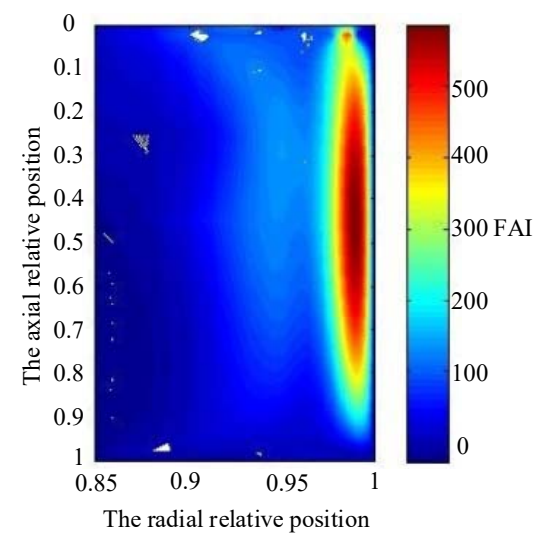

Fig. 7. The streamlines of the wall thermal drive quantity with 0.03 .

From Fig. 5 to Fig. 7, when the wall thermal drive quantity is 0.01 , a vortex is produced near the wall, but it is not intense. When the drive quantity increases to 0.02 , the flow function value at the center of the vortex is greater than other values around it, indicating that the flow of the vortex is intensified. When the drive quantity increases to 0.03 , the size and range of the vortex II remain the same. But the region of the vortex I decreases obviously and the vortex flow slows down. Meanwhile conversely, the other vortex regions remain unchanged. All of those is indicated that the increase of the wall thermal drive has an impact on certain drives, which weakens the axial circulation and increases the adverse effect. Through comparison and analysis, it is inferred that the most favorable wall thermal drive quantity to the axial circulation of the gas inside the rotor is 0.02 , and the value is not better as large as possible.

\section{Conclusion}

In this paper, the finite difference method is used to calculate the fluid parameters under the linear distribution of the wall temperature. Through the analysis of the streamlines, when the wall thermal drive quantity is 0.02 , the generation mechanism of several main vortexes in the flow field is obtained. By comparing the streamlines of different wall thermal drives, it is concluded that the wall thermal drive quantity with 0.02 is the most favorable for the axial circulation. Through this conclusion, it is possible to better control the axial circulation of the gas in the highspeed rotor, making it more conducive to the flow of the gas in the flow field, so as to better control the physical quantity of the gas at the outlet.

The temperature distribution of the wall is assumed to be linear and close to the ideal distribution in the calculation process in this paper. While the temperature of the wall in the actual high-speed rotating rotor is not linear affected by external factors, the next step will continue to calculate and analyze the flow field under different temperature distributions of the wall, especially the non-linear temperature distribution, which can be better adapt to the actual situation..

\section{References}

1. J.P.Lahargue, Soubbaramayer, J. Computer Meth. Appl. Mech. Eng 15 (1978)

2. Junni L, Xiuping L, Youzhi L, Mach. Des. Manu 2, 7-11 (2013)

3. Mengting Jiang, Bo Wang, J. Environ. Eng 6 (2012)

4. Weixing H, Jianming L, Zeyi X, Engineering Fluid Mechanics, 136-140 (2008)

5. Baoguo W, Xiang L, Weiguang H, J. Areo. Pow 25, 964-980 (2010)

6. Zhonghua L, Zhihui L, Haiyan L, Acta Aero. Sinica 31, 282-287 (2013)

7. Jhon D.Anderson, Computation Fluid Dynamics, 51-55 (2007)

8. H.G.Wod, G.Sanders, J. Fluid. Mech 127 (1983) 